\title{
Separation of Variables and the Computation of Fourier Transforms on Finite Groups, II
}

\author{
David Maslen $^{1}$, Daniel N. Rockmore $\|^{2}$, and Sarah Wolff $\equiv^{\text {" }}$ \\ ${ }^{1}$ HBK Capital Management, New York, NY 10036, \\ ${ }^{2}$ Departments of Mathematics and Computer Science, Dartmouth College, Hanover, NH 03755 \\ ${ }^{3}$ Department of Mathematics and Computer Science, Denison University, Granville, OH 43023
}

\begin{abstract}
We present a general diagrammatic approach to the construction of efficient algorithms for computing the Fourier transform of a function on a finite group. By extending work which connects Bratteli diagrams to the construction of Fast Fourier Transform algorithms we make explicit use of the path algebra connection and work in the setting of quivers. In this setting the complexity of an algorithm for computing a Fourier transform reduces to path counting in the Bratelli diagram, and we generalize Stanley's work on differential posets to provide such counts. Our methods give improved upper bounds for computing the Fourier transform for the general linear groups over finite fields, the classical Weyl groups, and homogeneous spaces of finite groups.

Résumé. Nous présentons une approche schématique générale à la construction d'algorithmes efficaces pour le calcul de la transformée de Fourier d'une fonction sur un groupe fini. En étendant le travail qui relie diagrammes Bratelli à la construction d'algorithmes efficaces nous faisons usage explicite de la connexion de l'algèbre des chemins et le travail dans le cadre de carquois. Dans ce cadre la complexité d'un algorithme de calcul de transformée de Fourier réduit à des comptages de certains sous-carquois du diagramme Bratelli. Nos méthodes donnent améliorées limites supérieures pour le calcul de la transformée de Fourier pour les groupes linéaires généraux sur les corps finis, les groupes de Weyl classiques, et des espaces homogènes de groupes finis.
\end{abstract}

Keywords. Fast Fourier Transform, Bratteli diagram, path algebra, quiver

\section{Introduction}

The Fast Fourier Transform (FFT) remains among the most important family of algorithms in information processing [Roc00]. It efficiently computes the discrete Fourier transform (DFT) which is equivalent to the matrix-vector multiplication

$$
\left(e^{2 \pi i j k / n}\right)_{j, k} \vec{f}
$$

for $i=\sqrt{-1}, j, k=0, \ldots n-1$, and $\vec{f}$ a complex-valued vector of length $n$ [Roc00]. This calculation can be framed in a number of ways. We take a representation theoretic point of view and cast the DFT as a

\footnotetext{
${ }^{\dagger}$ The second author was partially supported by AFOSR Award FA9550-11-1-0166 and the Neukom Institute for Computational Science at Dartmouth College

¥ The third author was partially supported by an NSF Graduate Fellowship under Grant No. DGE-1313911

1365-8050 @ 2016 Discrete Mathematics and Theoretical Computer Science (DMTCS), Nancy, France
} 
change of basis in $\mathbb{C}\left[C_{N}\right]$, the complex group algebra of the cyclic group of order $N$, from a natural basis of group element indicator functions to a basis of irreducible matrix elements. This perspective suggests a generalization of the DFT to finite nonabelian groups $G$ as the computation of a change of basis in $\mathbb{C}[G]$ from a basis of indicator functions to a basis of irreducible matrix elements and raises attendant questions of computational complexity addressed herein.

Let $T_{G}(R)$ denote the computational complexity of the Fourier transform on a group $G$ at a set of inequivalent irreducible representations $R$. Then $C(G)$ denotes the complexity of the group $G$, defined as

$$
C(G):=\min _{R}\left\{T_{G}(R)\right\} .
$$

For $N$ a "highly composite" number, Cooley and Tukey in 1965 famously presented an algorithm to show $C(\mathbb{Z} / N \mathbb{Z}) \leq O\left(N \log _{2} N\right)[\mathrm{CT} 65]$. Yavne [Yav68] later showed that for $N=2^{m}, C(\mathbb{Z} / N \mathbb{Z}) \leq$ $\frac{8}{3} N \log _{2} N-\frac{16}{9} N-\frac{2}{9}(-1)^{\log _{2}(N)}+2$. More recently, Johnson and Frigo [JF07] and Lundy and Van Buskirk [LVB07] further reduced the total number of complex multiplications required, but without affecting the overall group complexity $C(\mathbb{Z} / N \mathbb{Z})$. More generally, for $A$ an abelian group of size $N$, various efficiencies can be combined to prove the complexity of the DFT on $A$ is bounded above by $O\left(N \log _{2} N\right)$ [Dia80]. The deep and ongoing study of this problem has been motivated by a wide range of applications in digital signal processing and beyond (see e.g. [AT79, DMT13, TAL97]).

The Cooley-Tukey algorithm is undoubtedly the most famous of the FFTs. It is a divide-and-conquer algorithm whose key step is to rewrite the DFT on a cyclic group $C_{N}$ as a linear combination of DFTs on $C_{n}<C_{N}$ (for $n \mid N$ ). Iterating this step for a chain of subgroups of $C_{N}$ yields algorithms more efficient than a direct matrix-vector multiplication.

In this paper we continue a line of work that generalizes this approach to nonabelian groups [MR95, MR97b, MR00]. The computations are encoded via paths in a Bratteli diagram associated to the group of interest, which in turn means that matrix elements correspond to pairs of paths in the diagram, which for a given group element may only be nonzero when of a particular form. The "repeated units" of our divideand-conquer approach are subgraphs of a Bratteli diagram and efficiencies are gained by recognizing their multiple appearances in the corresponding calculation. This is the guts of the "separation of variables" (SOV) approach first introduced in [MR97b] and extended in [Mas98].

We take on the problem of laying a proper axiomatic and logical foundation for this approach and in so doing also produce improved algorithms for the important families of classical Weyl groups $B_{n}$ and $D_{n}$ and the general linear groups over finite fields $G L_{n}\left(\mathbb{F}_{q}\right)$ :

Theorem 1.1 $C\left(B_{n}\right) \leq n(2 n-1)\left|B_{n}\right|$.

Theorem $1.2 C\left(D_{n}\right) \leq \frac{n(13 n-11)}{2}\left|D_{n}\right|$.

Theorem 1.3 $C\left(G l_{n}\left(\mathbb{F}_{q}\right)\right) \leq\left(\frac{4^{n} q^{n+1}-q}{4 q-1}+\frac{n(n+1)(2 n+1)(q-1)}{6}\right)\left|G l_{n}(q)\right|$.

Improvements for the complexity of Fourier transforms on related homogeneous spaces are also gained. For example, let $B_{n} / B_{n-k}$ denote the homogenous space of the Weyl group $B_{n}$.

Theorem 1.4 $C\left(B_{n} / B_{n-k}\right) \leq k(4 n-2 k-1) \frac{\left|B_{n}\right|}{\left|B_{n-k}\right|}$.

The following is an extended abstract of [MRW]. Although we have made an effort to keep it selfcontained, full details can be found in the full paper. In Sections 2 and 3 we outline the preliminaries 
needed for our results, including a discussion of the main ideas behind the SOV approach. Our main theorem of Section 3, Theorem 3.5, reduces complexity counts to combinatorial path-counting. We conclude in Section 3.1 by generalizing Stanley's work on differential posets [Sta88, Sta90] to provide the main combinatorial counts needed for Theorems 1.1, 1.2, 1.3 , and 1.4

\section{Background}

\subsection{Fourier transforms and the group algebra}

Definition 2.1 Let $G$ be a finite group and $f$ a complex-valued function on $G$.

(i) Let $\rho$ be a matrix representation of $G$. Then the Fourier transform of $\mathbf{f}$ at $\rho$, denoted $\hat{f}(\rho)$, is the matrix sum

$$
\hat{f}(\rho)=\sum_{s \in G} f(s) \rho(s) .
$$

(ii) Let $R$ be a set of matrix representations of $G$. Then the Fourier transform of $\mathbf{f}$ on $\mathbf{R}$ is the direct sum of Fourier transforms of $f$ at the representations in $R$ :

$$
\mathcal{F}_{R}(f)=\bigoplus_{\rho \in R} \hat{f}(\rho) \in \bigoplus_{\rho \in R} \operatorname{Mat}_{\operatorname{dim} \rho}(\mathbb{C})
$$

When we compute the Fourier transform for a complete set of inequivalent irreducible representations $R$ of $G$ we refer to the calculation as the computation of a Fourier transform on $G$ (with respect to $R$ ).

Definition 2.2 Let $G$ be a finite group, $R$ a set of matrix representations of $G$.

(i) A straight-line program is a list of instructions for performing the operations $\times, \div,+,-$ on inputs and precomputed values.

(ii) The arithmetic complexity of a Fourier transform on $R$, denoted $T_{G}(R)$, is the minimum number of complex arithmetic operations needed to compute the Fourier transform of $f$ on $R$ via a straight-line program for an arbitrary complex-valued function $f$ defined on $G$. As in [Mas98] we will always define the arithmetic complexity to be the maximum of the number of complex multiplications and the number of complex additions.

(iii) The complexity of the group $G$, denoted $C(G)$ is defined by

$$
C(G):=\min _{R}\left\{T_{G}(R)\right\},
$$

where $R$ varies over all complete sets of inequivalent irreducible representations of $G$.

(iv) The reduced complexity, denoted $t_{G}(R)$, is defined by

$$
t_{G}(R)=\frac{1}{|G|} T_{G}(R)
$$


Let $\rho_{1}, \ldots, \rho_{m}$ be a complete set of inequivalent irreducible matrix representations of a group $G$ of dimensions $d_{1}, \ldots, d_{m}$, respectively. A direct computation of a Fourier transform would require at most $|G| \sum d_{i}^{2}=|G|^{2}$ arithmetic operations. Rewriting, for a direct computation we have

$$
C(G) \leq T_{G}(R) \leq|G|^{2}
$$

Fast Fourier transforms (FFTs) are algorithms for computing Fourier transforms that improve on this naive upper bound.

The group algebra $\mathbb{C}[G]$ is the space of all formal complex linear combinations of group elements under the product

$$
\left(\sum_{s \in G} f(s) s\right)\left(\sum_{t \in G} h(t) t\right)=\sum_{s, t \in G} f(s) h(t) s t .
$$

Elements of $\mathbb{C}[G]$ are in one-to-one correspondence with complex-valued functions on $\mathrm{G}$, and the group algebra product corresponds to convolution of functions.

A complete set $R$ of inequivalent irreducible matrix representations of a group $G$ determines a basis for $\mathbb{C}[G]$ and in this case the Fourier transform of a function $f$ on $G$ with respect to a complete set of inequivalent irreducible representations $R$ of $G$ is an algebra isomorphism

$$
\mathbb{C}[G] \stackrel{\mathcal{F}_{R}}{\longrightarrow} \bigoplus_{\rho \in R} M_{\operatorname{dim}(\rho)}(\mathbb{C}),
$$

and so as in [Cla89, Mas98]:

Lemma 2.3 The computation of the Fourier transform of a function $f$ on $G$ with respect to a complete set of irreducible representations $R$ is equivalent to computation of

$$
\sum_{s \in G} f(s) s
$$

in the group algebra, relative to a fixed basis for $R$.

\subsection{Bratteli diagrams and quivers}

The fundamental idea of the SOV approach is a recasting of the Cooley-Tukey algorithm in terms of graded quivers, which is an elaboration of path algebras derived from Bratteli diagrams.

Definition 2.4 A quiver $Q$ is a directed multigraph with vertex set $V(Q)$ and edge set $E(Q)$. For an arrow $e \in E(Q)$ from vertex $\beta$ to vertex $\alpha$, we call $\alpha$ the target of $e$ and $\beta$ the source of $e$.

Let $Q$ be a quiver. For each $e \in E(Q)$, let $t(e)$ denote the target of $e$ and $s(e)$ the source of $e$.

Definition 2.5 A quiver $Q$ is graded if there is a function $g r: V(Q) \rightarrow \mathbb{N}$ such that $\operatorname{gr}(t(e))>\operatorname{gr}(s(e))$, for each $e \in E(Q)$.

Example 2.6 Figure 1 is an example of a graded quiver. Each vertex $v$ is labeled by its grading, $\operatorname{gr}(v)$. 


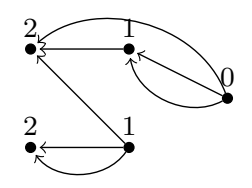

Fig. 1: A graded quiver

Definition 2.7 A Bratteli diagram is a finite graded quiver such that:

(i) there is a unique vertex with grading 0 , called the root,

(ii) if $v \in V(Q)$ is not the root then $v$ is the target of at least one arrow,

(iii) if $v \in V(Q)$ does not have grading of maximum value then $v$ is the source of at least one arrow,

(iv) for each $e \in E(Q), \operatorname{gr}(t(e))=1+\operatorname{gr}(s(e))$.

Example 2.8 Note that the quiver of Figure 1 is not a Bratteli diagram. However, by removing the top arrow and adding an arrow from vertex 0 to the bottom vertex of grading 1 , we produce a Bratteli diagram.

Consider a group algebra chain $\mathbb{C}\left[G_{n}\right]>\mathbb{C}\left[G_{n-1}\right]>\cdots>\mathbb{C}\left[G_{1}\right]>\mathbb{C}\left[G_{0}\right]=\mathbb{C}$. To associate a Bratelli diagram to this chain we follow the language of [Ram97]. Let $\rho$ be an irreducible representation of $G_{i}$, i.e., an irreducible $\mathbb{C}\left[G_{i}\right]$-module. Upon restriction to $G_{i-1}, \rho \downarrow_{G_{i-1}}$ decomposes as a direct sum of irreducible $\mathbb{C}\left[G_{i-1}\right]$-modules. For $\gamma$ an irreducible representation of $G_{i-1}$, let $M(\rho, \gamma)$ denote the multiplicity of $\gamma$ in $\rho \downarrow_{G_{i-1}}$.

Definition 2.9 For a chain of group algebras $\mathbb{C}\left[G_{n}\right]>\mathbb{C}\left[G_{n-1}\right]>\cdots>\mathbb{C}\left[G_{0}\right]$, the associated Bratteli diagram consists of vertices of grading $i$ labeled by the (equivalence classes of) irreducible representations of $G_{i}$, and each vertex labeled by an irreducible representation $\gamma$ of $G_{i-1}$ is connected to a vertex labeled by an irreducible representation $\rho$ of $G_{i}$ by $M(\rho, \gamma)$ arrows.

Example 2.10 Figure 2 shows two examples of Bratteli diagrams, with the gradings listed at the top. On the left we see the Bratteli diagram for a chain of group algebras for $C_{6}$ while on the right we see the Bratteli diagram for a chain of group algebras for the symmetric group $S_{3}$, viewing $S_{i}$ as the subgroup of $S_{n}$ that fixes the elements $\{i+1, \ldots, n\}$. Note that we distinguish $\mathbb{C}\left[S_{1}\right]$ from $\mathbb{C}$ only so that vertices at level $i$ correspond to representations of $\mathbb{C}\left[S_{i}\right]$.

For the group algebra $\mathbb{C}\left[C_{N}\right]$, irreducible representations are naturally indexed at each level by the integers $0, \ldots, N-1$, while for $\mathbb{C}\left[S_{n}\right]$, the irreducible representations are indexed by partitions of $n$. 

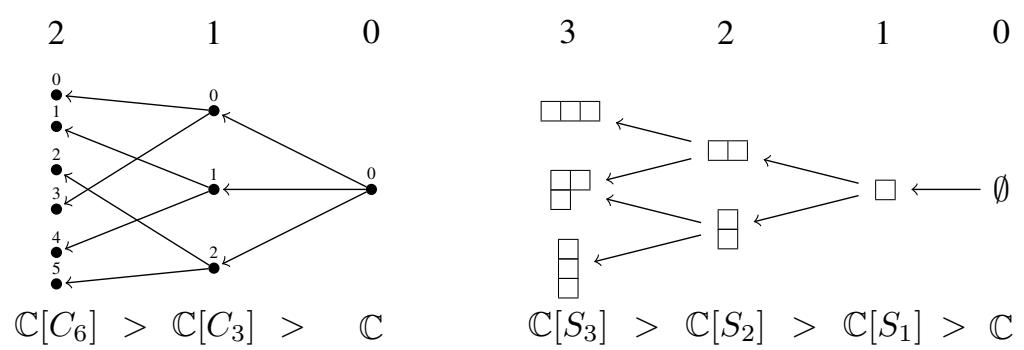

Fig. 2

Given a Bratteli diagram $\mathcal{B}$, there is a canonical chain of algebras associated to $\mathcal{B}$ called the chain of path algebras.

Definition 2.11 Let $\mathcal{B}$ be a Bratteli diagram. The path algebra (at level $\boldsymbol{i}$ ), denoted $\mathbb{C}\left[\mathcal{B}_{i}\right]$, is the $\mathbb{C}$-vector space with basis given by ordered pairs of paths of length $i$ in $\mathcal{B}$ which start at the root and end at the same vertex at level $i$.

Example 2.12 For the Bratteli diagram $\mathcal{B}$ of Figure 2 associated to the chain $\mathbb{C}\left[S_{3}\right]>\mathbb{C}\left[S_{2}\right]>\mathbb{C}\left[S_{1}\right]>$ $\mathbb{C}$, let $P_{1}, P_{2}, P_{3}, P_{4}$ be the paths from the root to level 3 in $\mathcal{B}$, labeled from top to bottom. Then the path algebra $\mathbb{C}\left[\mathcal{B}_{3}\right]$ has basis $\left\{\left(P_{1}, P_{1}\right),\left(P_{2}, P_{2}\right),\left(P_{2}, P_{3}\right),\left(P_{3}, P_{2}\right),\left(P_{3}, P_{3}\right),\left(P_{4}, P_{4}\right)\right\}$.

Note that for a vertex $v$, labeled by a representation $\rho$, the dimension of $\rho$ is given by the number of paths from the root to $v$.

Further, $\mathbb{C}\left[\mathcal{B}_{i}\right]$ embeds into $\mathbb{C}\left[\mathcal{B}_{i+1}\right]$ as a subalgebra by mapping any pair of paths $(P, Q) \in \mathbb{C}\left[\mathcal{B}_{i}\right]$ to the sum

$$
\sum_{e}(e \circ P, e \circ Q)
$$

over all arrows $e$ such that the source of $e$ is the target of $P$ (equivalently, of $Q$ ), and o denotes concatenation of paths. Thus, elements in these subalgebras are effectively determined by the initial "legs" of their paths. This is also equivalent to a choice of basis in the corresponding Wedderburn decomposition of the group algebra as a direct sum of matrix algebras, recognizing that for a given element, a number (equal to the total number of distinct paths that have the common middle "source" of tail of $P$ ) of irreducible matrix elements will take on the same value. Identification of this kind of common "unit" (formalized by the injection of one quiver into another) is the fundamental observation and technique of the quiver-based SOV approach.

Multiplication in the path algebra $\mathbb{C}\left[\mathcal{B}_{i}\right]$ linearly extends $(P, Q) *\left(P^{\prime}, Q^{\prime}\right)=\delta_{Q P^{\prime}}\left(P, Q^{\prime}\right)$ :

$$
\sum_{(P, Q)} a_{P Q}(P, Q) * \sum_{\left(P^{\prime}, Q^{\prime}\right)} b_{P^{\prime} Q^{\prime}}\left(P^{\prime}, Q^{\prime}\right)=\sum\left(\sum_{Q} a_{P Q} b_{Q Q^{\prime}}\right)\left(P, Q^{\prime}\right) .
$$

Lemma 2.13 Let $\mathbb{C}[G]=\mathbb{C}\left[G_{n}\right]>\mathbb{C}\left[G_{n-1}\right]>\cdots>\mathbb{C}\left[G_{1}\right]>\mathbb{C}\left[G_{0}\right]=\mathbb{C}$ be a chain of group algebras with Bratteli diagram $\mathcal{B}$. Then the chain of path algebras associated to $\mathcal{B}$ is isomorphic to the group algebra chain.

Lemma 2.13 allows for Lemma 2.3 to be recast in terms of elements of the path algebra. 


\section{The Separation of Variables Approach}

The first steps of the SOV approach involve expressing a path algebra element as a factorization over subsets of the Bratteli diagram in such a way as to disentangle the dependencies in the sum. To do so we first factor the Fourier transform through the subalgebras $\mathbb{C}\left[G_{i}\right]$. If we do this for a simple two-step chain, $\mathbb{C}[G]>\mathbb{C}[H]>\mathbb{C}$, we get a corresponding factorization

$$
\mathcal{F}:=\sum_{s \in G} f(s) \tilde{s}=\sum_{y \in Y} \sum_{h \in H} f(y h) \tilde{y} \tilde{h}=\sum_{y \in Y} \tilde{y} \sum_{h \in H} f(y h) \tilde{h}=\sum_{y \in Y} \tilde{y} F_{y},
$$

for $Y$ a set of coset representatives for $G / H$ such that for each $y \in Y$,

$$
F_{y}=\sum_{h \in H} f_{y}(h) \tilde{h} \in \mathbb{C}\left[\mathcal{B}_{H}\right]
$$

with $f_{y}(h):=f(y h)$.

This factorization allows us to obtain a simple, but key complexity estimate: given a set of coset representatives $Y$ for $G / H$ with $F_{y}$ (for each $y \in Y$ ) an arbitrary element of $\mathbb{C}\left[\mathcal{B}_{H}\right]$, define

$$
m_{G}(R, Y, H)=\frac{1}{|G|} \times\left\{\text { minimum number of operations required to compute } \sum_{y \in Y} \tilde{y} F_{y}\right\} .
$$

Then a restatement of Lemma 2.10 of [Mas98] and Proposition 1 of [DR90] gives

$$
t_{G}(R) \leq t_{H}\left(R_{H}\right)+m_{G}(R, Y, H) .
$$

This bound shows that to compute the Fourier transform of a complex function defined on $G$ we compute

$$
\mathcal{F}_{Y}:=\sum_{y \in Y} \tilde{y} F_{y}
$$

for $Y$ a set of coset representatives for $G / H$. In doing so, the complexity estimate "reduces" to a close study of the computation of $F_{Y}$.

The heart of the SOV approach is the efficient computation of $\mathcal{F}_{Y}$. It is comprised of three main steps:

1 Factor each term $\tilde{y} F_{y}$ and use these factorizations to rearrange $\mathcal{F}_{Y}$ into a recursive summation.

2 Each factor $x_{i}$ of each term corresponds to an element of the path algebra, and thus a particular subgraph of the Bratteli diagram. These subgraphs are given a vector space structure through an identification with a space of quiver morphisms.

3 By virtue of the vector space identification, the element multiplication $x_{i} x_{i+1}$ becomes a bilinear map whose complexity can be calculated directly in terms of the number of certain subgraphs in the Bratteli diagram.

To give the general idea, the "gluing" and summing operations that are multiplication in the path algebra mean that only certain kinds of "middle paths" contribute when two path algebra elements are multiplied. A complexity estimate thus becomes counting the number of subgraphs (subquivers) wherein this compatibility is respected - counting the occurrences of subquivers $\mathcal{Q}$ in the corresponding Bratteli diagram $\mathcal{B}$. Ultimately, this is the number of morphisms from $\mathcal{Q}$ into $\mathcal{B}$. We give a general example below. 


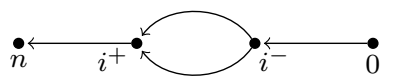

$Q_{i}$

Fig. 4

Example 3.1 Suppose $y \in \mathbb{C}[G]$ factors as $y=x_{1} x_{2}$ with $x_{i} \in \mathbb{C}\left[G_{i+2}\right] \cap$ Centralizer $\mathbb{C}\left[G_{i}\right]$. When we express $x_{i}$ in in the path algebra as $\tilde{x}_{i}=\sum_{(P, Q)}\left[x_{i}\right]_{P Q}(P, Q)$, an application of Schur's Lemma shows that $\left[x_{i}\right]_{P, Q}$ is 0 unless $P$ and $Q$ are paths in $\mathcal{B}$ that agree from level $n$ to level $i+1$, and from level $i-1$ to level 0 , as in the quivers $Q_{i}$ of the lefthand side of Figure 3 (see also [MR97al). The product $\tilde{x}_{1} \tilde{x}_{2}$ is indexed by a triple of paths resulting from gluing $Q_{2}$ to $Q_{1}$ by identifying the bottom path of $Q_{1}$ with the top path of $Q_{2}$, but maintaining the structures of $Q_{1}$ and $Q_{2}$ (the quiver on the righthand side of Figure 3). The complexity count is thus the careful counting of these compatible structures, which can be recast as the computation of the dimension of a space of quiver morphisms.
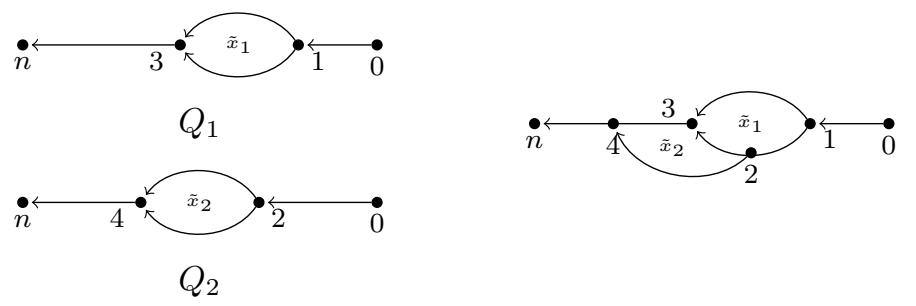

Fig. 3

For products with more factors we iterate this gluing process. Example 3.3 below gives further details. The SOV approach consists of factoring $\tilde{y} F_{y}$, forming the graph (akin to Fig. 3) and determining the subgraphs corresponding to each individual product.

Definition 3.2 For a path algebra product $x_{1} \cdots x_{m}$, let $i^{+}$denote the smallest integer such that $x_{i} \in$ $\mathbb{C}\left[\mathcal{B}_{i^{+}}\right]$and let $i^{-}$denote the largest integer less than or equal to $i^{+}$such that $x_{i} \in \operatorname{Centralizer}\left(\mathbb{C}\left[\mathcal{B}_{i^{-}}\right]\right)$. Then for $1 \leq i \leq m$ define

$$
X_{i}:=\mathbb{C}\left[\mathcal{B}_{i^{+}}\right] \cap \operatorname{Centralizer}\left(\mathbb{C}\left[\mathcal{B}_{i^{-}}\right]\right) .
$$

To each space $X_{i}$, associate the quiver $Q_{i}$ of Figure 4 Note that $Q_{i}$ is the quiver associated to every element of $X_{i}$. We show in the full paper [MRW] that $X_{i}$ has dimension equal to the number of occurrences of $Q_{i}$ in the Bratteli diagram $\mathcal{B}$. Denote this number by \# $\operatorname{Hom}\left(Q_{i} ; \mathcal{B}\right)$. An "occurrence" of $Q_{i}$ is the same as an injective map from $Q_{i}$ into $\mathcal{B}$. Thus, $\# \operatorname{Hom}\left(Q_{i} ; \mathcal{B}\right)$ is also the dimension of this space of morphisms of $Q_{i}$ into $\mathcal{B}$.

In this setting (bilinear) group algebra multiplication is transformed into a bilinear map on products of associated spaces of quiver morphisms. Call this map *. As the notation and details are more technical than illuminating, we defer the explicit definition of $*$ and discussion of its properties to the full paper. However, even with deferring this we can present an example and the final algorithm. 

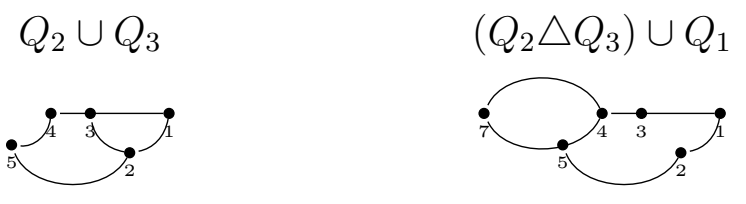

Fig. 6

Example 3.3 Suppose $\tilde{y}=x_{1} x_{2} x_{3}$, with

$$
\begin{array}{ll}
1^{+}=7, & 1^{-}=4, \\
2^{+}=3, & 2^{-}=1, \\
3^{+}=5, & 3^{-}=2 .
\end{array}
$$

Figure 5 shows the quivers $Q_{i}$ and the quiver $\mathcal{Q}$ formed by gluing $Q_{1}$ to $Q_{2}$ to $Q_{3}$.
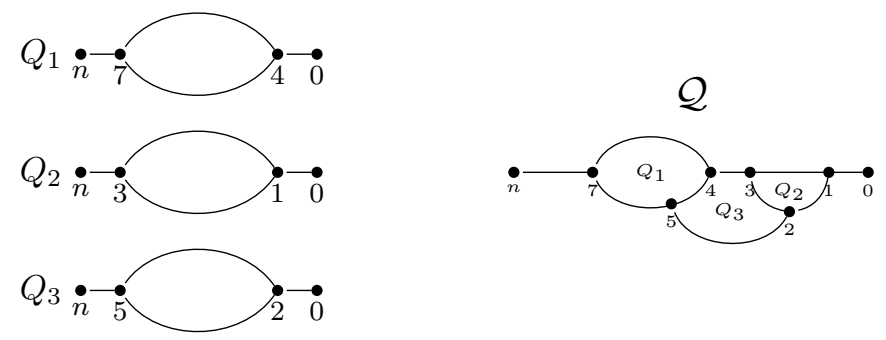

Fig. 5

Translated to the space of quiver morphisms, the complexity of computing $x_{1} x_{2} x_{3}$ in the group algebra becomes the complexity of computing $w_{1} * w_{2} * w_{3}$, where $w_{i}$ is in the space of morphisms from $Q_{i}$ to $\mathcal{B}$. In this space, the complexity of computing $w_{1} * w_{2} * w_{3}$ is equivalent to the complexity of computing $w_{\sigma(1)} * w_{\sigma(2)} * w_{\sigma(3)}$, for $\sigma \in S_{3}$.

For example, for $\sigma=(123)$, we determine the complexity of $w_{2} * w_{3} * w_{1}$. The complexity of $w_{2} * w_{3}$ is \# $\operatorname{Hom}\left(Q_{2} \cup Q_{3} ; \mathcal{B}\right)$, where $Q_{2} \cup Q_{3}$ is as in Figure 6 , the subquiver of $\mathcal{Q}$ corresponding to $Q_{2}$ and $Q_{3}$ (in Figure 6 we need only consider the subquiver formed by the segments of $Q_{2} \cup Q_{3}$ where not all three of the paths agree). The complexity of $\left(w_{2} * w_{3}\right) * w_{1}$ is \# $\operatorname{Hom}\left(\left(Q_{2} \triangle Q_{3}\right) \cup Q_{1} ; \mathcal{B}\right)$, where $Q_{2} \triangle Q_{3}$ is the quiver of Figure 6 associated to the space containing $w_{2} * w_{3}$. Note that as per the notation $Q_{2} \triangle Q_{3}$ is in fact the symmetric difference of $Q_{2}$ and $Q_{3}$, i.e., the edges of $Q_{2} \cup Q_{3}$ not in $Q_{2} \cap Q_{3}$.

Lemma 3.4 For $Q_{i}$ (respectively, $Q_{j}$ ) the quiver associated to $X_{i}$ (respectively, $X_{j}$ ), $x_{i} * x_{j}$ requires at most \# $\operatorname{Hom}\left(Q_{i} \cup Q_{j} ; \mathcal{B}\right)$ scalar multiplications and fewer additions.

With Lemma 3.4 we now have our main general result. Choose $m \in \mathbb{N}$ and a subset $X \subseteq\left(\mathbb{C}\left[\mathcal{B}_{n}\right]\right)^{m}=$ $\mathbb{C}\left[\mathcal{B}_{n}\right] \times \cdots \mathbb{C}\left[\mathcal{B}_{n}\right]$ such that $|X|=|Y|$ and for each $y \in Y$ there exists $\left(x_{1}, \ldots, x_{m}\right) \in X$ with $\tilde{y} F_{y}=$ $x_{1} \cdots x_{m}$. Thus, $X$ can be thought of as a choice of factorization into at most $m$ elements of each term $\tilde{y} F_{y}$. 
Theorem 3.5 For $x_{i}$ as above and $\sigma \in S_{m}$, let $Q_{i}^{\sigma}$ denote the quiver associated to $w_{i}=x_{\sigma(i)}$. Let $W_{i}=\left\{\left(w_{i+1}, \ldots w_{m}\right) \mid\left(x_{1} \ldots x_{m}\right) \in X\right\}$. Then we may compute $\sum_{y \in Y} \tilde{y} F_{y}$ in at most

$$
\sum_{i=1}^{m-1}\left|W_{i-1}\right| \# \operatorname{Hom}\left(\left(Q_{1}^{\sigma} \triangle \cdots \triangle Q_{i}^{\sigma}\right) \cup Q_{i+1}^{\sigma} ; \mathcal{B}\right)
$$

multiplications and fewer additions.

\subsection{Morphisms into Bratteli Diagrams}

Theorem 3.5 reduces complexity counts to determining \# $\operatorname{Hom}(Q ; \mathcal{B})$ for $Q$ a quiver as in Example 6 and $\mathcal{B}$ a Bratteli diagram. For Bratteli diagrams associated to group algebra chains we generalize Stanley's work on differential posets [Sta88] to deterime these counts.

Let $\mathbb{C}[V(\mathcal{B})]$ denote the space of finitely supported linear combinations of vertices of $\mathcal{B}$, let $\mathcal{B}^{i}$ denote the vertices $\alpha \in V(\mathcal{B})$ with $\operatorname{gr}(\alpha)=i$, and let $\mathbb{C}\left[\mathcal{B}^{i}\right]$ denote the space of finitely supported linear combinations of vertices at level $i$ in $\mathcal{B}$. Define an inner product $\langle$,$\rangle on \mathbb{C}[V(\mathcal{B})]$ making the vertices orthonormal. For $\alpha, \beta \in V(B)$, let $M_{B}(\alpha, \beta)$ denote the number of paths from $\beta$ to $\alpha$ in $B$. Note that because $\mathcal{B}$ is a Bratteli diagram, $\alpha, \beta \in V(\mathcal{B})$ correspond to irreducible representations $\gamma, \rho$ and $M_{\mathcal{B}}(\alpha, \beta)=M(\gamma, \rho)$, as in Definition 2.9 .

As in [Sta88] define linear operators $U$ and $D$ on $\mathbb{C}[V(\mathcal{B})]$ by linearly extending the action on $\alpha \in \mathcal{B}^{i}$ :

$$
\begin{aligned}
U \alpha & =\sum_{\gamma \in \mathcal{B}^{i+1}} M_{\mathcal{B}}(\gamma, \alpha) \gamma, \\
D \alpha & =\sum_{\beta \in \mathcal{B}^{i-1}} M_{\mathcal{B}}(\alpha, \beta) \beta,
\end{aligned}
$$

where, by convention, if $\mathcal{B}$ has highest grading $n, \mathcal{B}^{-1}=\emptyset=\mathcal{B}^{n+1}=\mathcal{B}^{n+2}=\cdots$.

Note 3.6 As the vertices of $\mathcal{B}$ are labeled by the irreducible representations of $\mathbb{C}\left[\mathcal{B}_{i}\right]$, elements of $\mathbb{C}\left[\mathcal{B}^{i}\right]$ correspond to representations of the path algebra $\mathbb{C}\left[\mathcal{B}_{i}\right]$. In this context, $U$ is induction and $D$ restriction (see [GdlHJ89] Proposition 2.3.1).

Let $d_{\alpha}$ denote the dimension of the representation corresponding to vertex $\alpha$. Theorem 3.8 below generalizes Theorem 3.7 of [Sta88] and Theorem 2.3 of [Sta90].

Definition 3.7 Let $w=w_{l} \cdots w_{1}$ be a word in $U$ and $D$ and let $\mathcal{S}=\left\{i \mid w_{i}=D\right\}$. For each $i \in \mathcal{S}$, let $a_{i}=\#\left\{D\right.$ 's in $w$ to the right of $\left.w_{i}\right\}$, and similarly let $b_{i}=\#\left\{U\right.$ 's in $w$ to the right of $\left.w_{i}\right\}$. If $b_{i}-a_{i} \geq 0$ for all $i \in \mathcal{S}$, we call $w$ an admissible word.

Theorem 3.8 Let $\mathcal{B}$ be the Bratteli diagram associated to a group algebra chain and let $\lambda_{i}=\left|G_{i} / G_{i-1}\right|$. Then for $w=D^{d_{n}} U^{u_{n}} \cdots D^{d_{1}} U^{u_{1}}$ an admissible word in $U$ and $D, s=\sum_{i=1}^{n} u_{i}-d_{i}$, and $\alpha \in \mathcal{B}^{s}$,

$$
\langle w \hat{0}, \alpha\rangle=d_{\alpha} \prod_{i \in \mathcal{S}} \lambda_{b_{i}-a_{i}} .
$$

Definition 3.9 A quiver $Q$ is $\boldsymbol{n}$-toothed if it consists of $2 n+1$ (not necessarily distinct) vertices $\gamma_{0}, \ldots, \gamma_{n}$, $\beta_{1}, \ldots, \beta_{n}$ and distinct arrows connecting $\gamma_{i-1}$ to $\beta_{i}$ and $\gamma_{i}$ to $\beta_{i}$. 


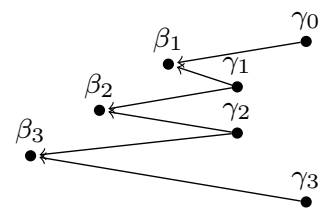

Fig. 7

Example 3.10 The quiver of Figure 7 is an example of a 3-toothed quiver.

Theorem 3.11 Let $\mathcal{B}$ be a locally free Bratteli diagram, $Q$ an $n$-toothed quiver with vertices $\gamma_{i}$ at level $l_{i}, \beta_{i}$ at level $m_{i}$. Then for $w=D^{m_{n}-l_{n}} U^{m_{n}-l_{n-1}} \ldots D^{m_{1}-l_{1}} U^{m_{1}-l_{0}}$,

$$
\# \operatorname{Hom}(Q ; \mathcal{B})=\sum_{\alpha \in \mathcal{B}^{l_{n}-l_{0}}}\langle w \hat{0}, \alpha\rangle=\prod_{i \in \mathcal{S}} \lambda_{b_{i}-a_{i}} \sum_{\alpha \in \mathcal{B}^{l_{n}}-l_{0}} d_{\alpha} .
$$

Proof: Follows from Theorem 3.8 and induction.

Theorem 3.11 together with Theorem 3.5 provides the complexity results of Theorems 1.1, $1.2,1.3$

\section{References}

[AT79] L. Auslander and R. Tolimieri. Is computing with the finite Fourier transform pure or applied mathematics? Bull. Amer. Math. Soc. (N.S.), 1(6):847-897, 1979.

[Cla89] M. Clausen. Fast generalized Fourier transforms. Theoret. Comput. Sci., 67(1):55-63, 1989.

[CT65] J. Cooley and J. Tukey. An algorithm for the machine calculation of complex Fourier series. Math. Comp., 19:297-301, 1965.

[Dia80] P. Diaconis. Average running time of the fast Fourier transform. J. Algorithms, 1:187-208, 1980.

[DMT13] A. Danelakis, M. Mitrouli, and D. Triantafyllou. Blind image deconvolution using a banded matrix method. Numer. Algorithms, 64(1):43-72, 2013.

[DR90] P. Diaconis and D. Rockmore. Efficient computation of the Fourier transform on finite groups. J. Amer. Math. Soc., 3(2):297-332, 1990.

[GdlHJ89] F. Goodman, P. de la Harpe, and V. Jones. Coxeter Graphs and Towers of Algebras, volume 14 of Mathematical Sciences Research Institute Publications. Springer-Verlag, New York, 1989.

[JF07] S. Johnson and M. Frigo. A modified split-radix FFT with fewer arithmetic operations. IEEE Trans. Signal Process., 55(1):111-119, 2007.

[LVB07] T. Lundy and J. Van Buskirk. A new matrix approach to real FFTs and convolutions of length $2^{k}$. Computing, 80(1):23-45, 2007. 
[Mas98] D. Maslen. The efficient computation of Fourier transforms on the symmetric group. Math. Comp., 67(223):1121-1147, 1998.

[MR95] D. Maslen and D. Rockmore. Adapted diameters and FFTs on groups. In Proc. 6th ACMSIAM SODA, pages 253-262. ACM, 1995.

[MR97a] D. Maslen and D. Rockmore. Generalized FFTs-a survey of some recent results. In Groups and computation, II (New Brunswick, NJ, 1995), volume 28 of DIMACS Ser. Discrete Math. Theoret. Comput. Sci., pages 183-237. Amer. Math. Soc., Providence, RI, 1997.

[MR97b] D. Maslen and D. Rockmore. Separation of variables and the computation of Fourier transforms on finite groups. I. J. Amer. Math. Soc., 10(1):169-214, 1997.

[MR00] D. Maslen and D. Rockmore. Double coset decompositions and computational harmonic analysis on groups. Journal of Fourier Analysis and Applications, 6(4):349-388, 2000.

[MRW] David K. Maslen, Daniel N. Rockmore, and Sarah Wolff. Separation of variables and the computation of Fourier transforms on finite groups. II. preprint.

[Ram97] A. Ram. Seminormal representations of Weyl groups and Iwahori-Hecke algebras. Proc. London Math. Soc. (3), 75(1):99-133, 1997.

[Roc00] D. Rockmore. The FFT: An algorithm the whole family can use. Computing in Science and Eng., 2(1):60-64, January 2000.

[Sta88]Ｒ. Stanley. Differential posets. J. Amer. Math. Soc., 1(4):919-961, 1988.

[Sta90] R. Stanley. Variations on differential posets. In Invariant theory and tableaux (Minneapolis, MN, 1988), volume 19 of IMA Vol. Math. Appl., pages 145-165. Springer, New York, 1990.

[TAL97] R. Tolimieri, M. An, and C. Lu. Algorithms for Discrete Fourier Transform and Convolution. Signal Processing and Digital Filtering. Springer-Verlag, New York, second edition, 1997.

[Yav68] R. Yavne. An economical method for calculating the discrete Fourier transform. Proc. AFIPS Fall Joint Computer Conf., 33:115-125, 1968. 